\title{
Forecasting Site-Specific Leaf Wetness Duration for Input to Disease-Warning Systems
}

\author{
K. S. Kim, Sustainable Land Use, HortResearch, Private Bag 92169, Auckland, New Zealand; and M. L. Gleason, \\ Department of Plant Pathology, and S. E. Taylor, Department of Agronomy, Iowa State University, Ames 50011
}

\begin{abstract}
Kim, K. S., Gleason, M. L., and Taylor, S. E. 2006. Forecasting site-specific leaf wetness duration for input to disease-warning systems. Plant Dis. 90:650-656.

Empirical models based on classification and regression tree analysis (CART model) or fuzzy logic (FL model) were used to forecast leaf wetness duration (LWD) $24 \mathrm{~h}$ into the future, using site-specific weather data estimates as inputs. Forecasted LWD and air temperature then were used as inputs to simulate performance of the Melcast and TOM-CAST disease-warning systems. Overall, the CART and FL models underpredicted LWD with a mean error (ME) of 2.3 and $3.9 \mathrm{~h}^{-1} \mathrm{day}^{-1}$, respectively. The CFL model, a corrected version of the FL model using a weight value, reduced ME in LWD forecasts to $-1.1 \mathrm{~h} \mathrm{day}^{-1}$. In the Melcast and TOM-CAST simulations, the CART and CFL models predicted timing of occurrence of action thresholds similarly to thresholds derived from on-site weather data measurements. Both models forecasted the exact spray dates for approximately $45 \%$ of advisories derived from measurements. When hindcast and forecast estimates derived from site-specific estimates provided by SkyBit Inc. were used as inputs, the CART and CFL models forecasted spray advisories within 3 days for approximately $70 \%$ of simulation periods for the Melcast and TOM-CAST disease-warning systems. The results demonstrate that these models substantially enhance the accuracy of commercial site-specific LWD estimates and, therefore, can enhance performance of diseasewarning systems using LWD as an input.
\end{abstract}

Additional keywords: melon, tomato

Disease-warning systems are management decision aids that can reduce the frequency of fungicide or bactericide sprays by enabling growers to monitor the risk of disease epidemics on their farms $(6,18,19)$. Weather data are key inputs to most disease-warning systems. In part because of the challenges of gaining timely access to the local-scale weather data needed to operate warning systems (6), however, few of these systems have been implemented widely.

Although weather sensors and dataloggers located in crop canopies can generate input data for warning systems, this approach is impractical for many growers because it is too costly, laborious, or unreliable $(6,9,17)$. A potentially more practical way to acquire local (i.e., site-specific) weather data is to purchase "estimates" of these data. Site-specific weather data have been available from commercial providers in North America for more than a decade, and several attempts have been made to evaluate or enhance the suitability of this technology for warning system applications $(7,14,29)$.

Corresponding author: M. Gleason

E-mail:mgleason@iastate.edu

Accepted for publication 27 December 2005.

DOI: 10.1094/PD-90-0650

(C) 2006 The American Phytopathological Society
Timeliness of warning system advisories also affects their practical value. A spray advisory received after the start of several days of continuous rainfall, for example, may not be helpful if the rainfall prevents a grower from making a timely spray. Nearly all efforts to develop or implement warning systems have relied solely on hindcast weather data (i.e., data measured or estimated some hours or days in the past). Utilizing forecast weather data, on the other hand, potentially could enhance effectiveness of a warning system $(10,28,29)$. For example, advance warning of the disease risk level could be valuable against rapidly developing diseases such as fire blight of pome fruit (10), if available fungicides or bactericides lack postinfection activity, or when forecasted weather could preclude responding to a spray advisory in a timely manner.

Leaf wetness duration (LWD) is an important input to many disease-warning systems because the risk of epidemics of many foliar diseases is directly related to the duration of periods when free water is present on crop surfaces (9). Currently, several U.S. companies provide sitespecific estimates of air temperature, relative humidity, wind speed, and LWD, not only in hindcast mode but also as forecasts up to $72 \mathrm{~h}$ into the future. Forecasted weather estimates at site-specific scale (e.g., $1 \mathrm{~km}^{2}$ ) could offer growers two important benefits: convenient access to local weather data and the ability to anticipate disease outbreaks.

However, it is uncertain whether LWD forecasts are sufficiently accurate for use in disease-warning systems, in part because forecasted weather data include inherent error caused by extrapolation into the future. Furthermore, when forecasts are site-specific, additional errors are incurred due to spatial interpolation to a specific locality (7). It also may be difficult to forecast LWD accurately, because inaccurate weather data used to estimate LWD, such as relative humidity, air temperature, and wind speed, could result in misidentification of wetness occurrence $(8,14)$. In addition, the spatial variability of LWD makes it difficult to interpolate or extrapolate information to distant (e.g., $>30 \mathrm{~km}$ ) sites $(6,26)$.

Nevertheless, it may be possible to reduce warning-system errors using models that can estimate LWD accurately using site-specific weather forecasts. LWD estimation models have been developed using a wide range of approaches, including energy balance equations, neural networks, classification and regression trees, and fuzzy logic $(1,3,4,8,13,20,22)$. Physical models incorporate the energy balance principles that govern the occurrence of free water on a crop canopy. Some physical models can estimate LWD quite accurately (within $1 \mathrm{~h} \mathrm{day}^{-1} ; 22$ ), but their application is limited because they require input variables, such as cloud cover, that are not recorded at most standard weather stations. Physical models also are highly sensitive to inaccuracy in their input data (21). In contrast, empirical models, which are developed through statistical best-fit processes instead of, or in conjunction with, energy balance principles, may be less dependent on accuracy of input data, and require fewer and more readily accessible input variables (14).

The present study evaluated the suitability of empirical LWD models for providing site-specific, forecast weather estimates to implement disease-warning systems. The objectives were to quantify accuracy of LWD forecasts using empirical models and validate the LWD forecasts as simulated inputs to disease-warning systems for muskmelon and tomato.

\section{MATERIALS AND METHODS}

Weather data. Air temperature, relative humidity (RH), wind speed, and LWD 
measurements were recorded hourly at 15 standard weather stations in Iowa (IA), Illinois (IL), and Nebraska (NE) from May to September 1998 and 1999. Air temperature and RH were measured at a height of $1.5 \mathrm{~m}$. Wind speed was measured at $3 \mathrm{~m}$ in IA and NE and $10 \mathrm{~m}$ in IL. Electronic wetness sensors (Model 237; Campbell Scientific, Logan, UT) were deployed at a $45^{\circ}$ angle, facing north, on level, unobstructed sites above managed turfgrass, $0.3 \mathrm{~m}$ above the ground. These sensors were coated with latex paint to increase sensitivity to small water droplets and their accuracy was calibrated within $1 \mathrm{~h}$ day $^{-1}$ $(2,5,17,24)$. When wetness was detected for $\geq 30 \mathrm{~min}$ in an hour, the hour was counted as wet $(=1)$; otherwise, the hour was scored as dry $(=0)$.

Hourly, site-specific weather estimates (SkyBit Inc., Bellefonte, PA) $(7,11,12)$, obtained for the same sites and dates as the on-site weather measurements included hindcast estimates for the previous $24 \mathrm{~h}$ as well as forecasts for the subsequent $24 \mathrm{~h}$. These data were used as inputs to empirical models to obtain site-specific prediction of LWD.

LWD models. Three empirical models were used to predict LWD: the CART/SLD/Wind model (hereafter abbreviated as "CART model"; 8,14), the Fuzzy model ("FL model"; 13), and a corrected version of the Fuzzy model ("CFL model"; 15) (Table 1). The SkyBit wetness model (SkyBit model), whose LWD estimation algorithm is proprietary, also was included in our analysis.

CART model. The CART model uses a hierarchical decision tree based on threshold values of dew point depression, wind speed, and RH (8). The decision tree structure was constructed empirically to minimize errors in classifying hours during which leaves were wet or dry. Stepwise linear discriminant analysis also was used to determine wetness classification rules. Because the SkyBit dataset included wind speed estimated or forecasted at a height of $10 \mathrm{~m}$, these estimates or forecasts were corrected to wetness sensor height by applying the log profile of wind speed (14).

FL model. The FL model is an empirical model that inputs air temperature, $\mathrm{RH}$, and wind speed (13). Its variables and decision rules approximate occurrence of wetness rather than obtain an exact solution. The FL model translates a numerical value of an input variable (e.g., air temperature of $10^{\circ} \mathrm{C}$ ) into a qualitative term (e.g., "low" or "high") and processes logic statements, such as "If air temperature is high, then wetness is likely absent." The numerical output of the FL model ranges from 0 to 1, indicating wetness occurrence at $\geq 0.5$. For a more detailed explanation of this application of fuzzy logic, consult Kim et al. (13).

CFL model. When systematic errors in input data occurred, it was likely that output of the FL model would deviate from reality. Temporal and spatial errors incurred in spatial interpolation of weather data by SkyBit Inc. were assessed by Gleason et al. (7). To minimize influence of input errors on LWD model forecasts, a correction factor was applied to output of the FL model (15). The correction factor was determined through a training process using a data set that included the SkyBit hindcast estimates of weather variables obtained in 1998 and 1999. The training set was split arbitrarily into 10 subsets and root mean square error (RMSE) was calculated for each subset to represent different weather condition sets. A correction factor was chosen to be a $2.5 \%$ increment of the output of the FL model, at which RMSE of most subsets was minimized (K. S. Kim, unpublished data). The resulting model was termed the corrected fuzzy logic (CFL) model.

Analysis of forecasted weather data. Measurements obtained from wetness sensors at the weather stations were assumed to represent true LWD, and deviations of prediction by LWD models from those values were assumed to represent errors. Model accuracy was quantified for 24-h periods that began at noon to encompass entire dew-eligible periods. Mean error (ME) was calculated by averaging differences between measured and forecasted LWD. Mean absolute error (MAE) was computed by summing hours in which measured and forecasted LWD disagreed. ME provided a measure of the tendency to over- or underpredict LWD, whereas MAE assessed overall accuracy. Pedro and Gillespie (22) suggested that an estimation error within $1 \mathrm{~h}$ day $^{-1}$ was a reasonable goal for LWD estimation by physical models. However, we used an MAE of $2 \mathrm{~h} \mathrm{day}^{-1}$ as a threshold of acceptable accuracy for LWD forecasts because additional error from spatial interpolation and temporal extrapolation is likely to be inherent (21). In addition, correlations between measurements and forecasts were obtained to quantify errors in the SkyBit weather forecasts.

Application of forecasted weather data to disease-warning systems. In simulation trials, forecasted LWD and air temperature were used as inputs to the Melcast disease-warning system for melon anthracnose (16) and the TOM-CAST disease-warning system for several fungal diseases of tomato $(23,25)$. Melcast and TOM-CAST were chosen for testing in this study because they use LWD as an input and have been implemented widely by growers in North America. Melcast and TOM-CAST calculate the sum of daily disease-risk ratings, called "environmental favorability indices" (EFI) and "disease severity values" (DSV), respectively, and recommend application of a fungicide spray when the sum reaches an action threshold.

Values of warning-system indexes calculated from measured LWD and air temperature during the wetness periods were assumed to be true, and the accuracy of estimates calculated by forecasting models was assessed by comparing them to the "true" values. Because values of the disease-warning indexes are discrete, an expected value was calculated as follows:

$E(D W I=i)=\sum_{i=1}^{n} p(D W I=i) \cdot i$

where DWI is a disease-warning index whose value ranged from 0 to the largest value of DWI corresponding to either EFI or DSV $(n)$. The probability, $p$, for each index was expressed as:

$p(D W I=i)=\frac{N_{i}}{N}$

where $N_{i}$ and $N$ are number of days on which the value of DWI is equal to $i$ and total days, respectively. For example, suppose that a disease-warning index value of 1 was derived from forecasted weather data for each of 10 days, whereas index values of 0,1 , and 2 were derived from measured weather data on 2, 4 , and 4 days, respectively, during the same period. In this case, the value of the forecast index would be 1 , whereas the "true" value would be $1.2(=[0 \times 2 / 10]+[1 \times 4 / 10]+$ $[2 \times 4 / 10])$, indicating that the model underestimated "true" disease risk.

Forecasting strategy complemented with measured weather data. Forecast weather data would be most useful to growers on days when a warning system's action threshold could be attained. On other days, however, it would make more sense to derive warning system index values from hindcast data because it is inherently more reliable than forecast data. Measurements made at weather stations are the most reliable hindcast data, and local weather measurements potentially could be available to some growers; therefore, we used on-site measurements as one source of hindcast data in our simulations.

Table. 1. List of leaf wetness duration (LWD) models included in this study

\begin{tabular}{lccc}
\hline Model name & Acronym & Type $^{\mathbf{a}}$ & References $^{\text {Parical }}$ \\
\hline SkyBit wetness model & SkyBit & Physical & 8,14 \\
CART/SLD/Wind model & CART & Empirical & 7,14 \\
Fuzzy model & FL & Empirical & 13 \\
Corrected Fuzzy model & CFL & Empirical & 15 \\
\hline
\end{tabular}

a Base algorithms of LWD models are proprietary, classification and regression tree with step-wise linear discriminant analysis, and fuzzy logic system, for SkyBit, CART, and FL model, respectively. The CFL model is obtained from multiplying a factor to the output of the FL model. 
If a value of the cumulative index on a given day was less than the action threshold (35 for Melcast and 20 for TOM-CAST), the forecasted disease-warning index value was replaced by the index value calculated from hindcast measurements for that day. Spray dates were determined as dates on which the cumulative index attained or exceeded an action threshold.

Because occasional missing weather measurements made it impossible to accumulate continuous season-long indices for some sites, individual simulation periods were analyzed in our study. An individual simulation period was terminated when a spray advisory was issued. Periods with missing data were discarded, and accumulation of disease-warning index values was reinitiated on the next date that had complete data.

Forecasting strategy complemented with estimated hindcast data. Warning system spray advisories also can be determined by using site-specific hindcast weather esti-

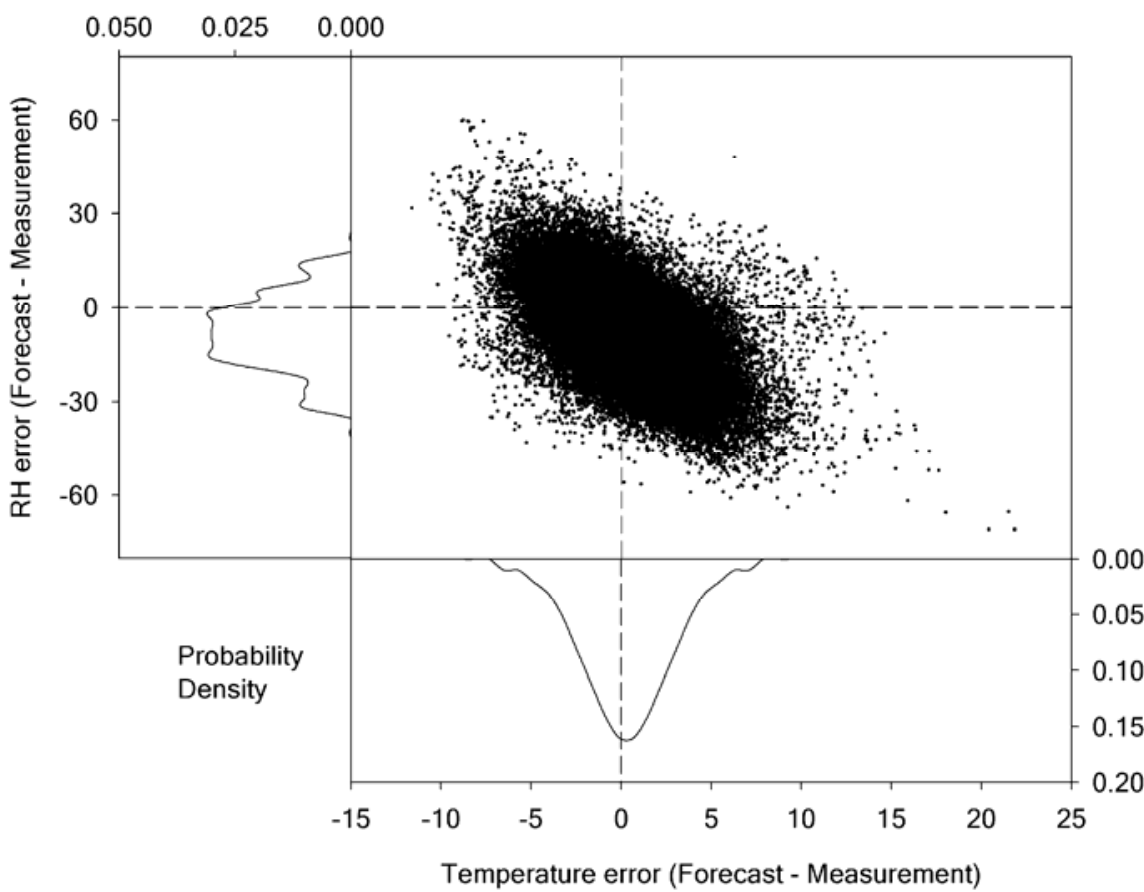

Fig. 1. Distribution of site-specific forecast error in estimating air temperature $\left({ }^{\circ} \mathrm{C}\right)$ and relative humidity (\%) at 15 sites in Iowa, Illinois, and Nebraska during 1998 and 1999. Site-specific weather forecasts were obtained from SkyBit Inc. Probability density indicates the frequency of data at a given error value. mates rather than on-site measurements. Because many growers lack access to onsite measurements, it could be practical for them to rely instead on forecast spray advisories calculated from site-specific hindcast weather estimates. First, LWD was estimated using the CART, FL, CFL, or SkyBit models. Mean air temperature also was calculated for the corresponding estimated wetness periods for each model, and values of disease-warning indexes were obtained from these data sets. Index values from hindcast estimates were summed to determine a cumulative disease-warning index. To determine a spray date, index values determined from forecasted data later were added to the cumulative index in the same manner as described above for measured weather data.

\section{RESULTS}

The $R^{2}$ values comparing variation between measured and forecasted data for air temperature and RH were 0.83 and 0.64 , respectively. Most SkyBit forecasts of air temperature were within $\pm 5^{\circ} \mathrm{C}$ of measured values, and the proportion of overestimates and underestimates was approximately equal (Fig. 1). In contrast, RH was more commonly underpredicted than overpredicted. The SkyBit forecasts often underestimated RH during hours in which air temperature was overestimated, presumably because overestimated air temperature resulted in overestimated saturated vapor pressure. However, there also were many hours in which RH was underpredicted even though air temperature was also underpredicted.

Overall, the CFL model predicted LWD within an ME of $-1.1 \mathrm{~h} \mathrm{day}^{-1}$, whereas the SkyBit, CART, and FL model underpredicted LWD by $>2 \mathrm{~h} \mathrm{day}^{-1}$ (Table 2). The

Table 2. Mean error (ME) and mean absolute error (MAE) for forecasted leaf wetness duration (LWD) at 15 sites in Iowa (IA), Illinois (IL), and Nebraska (NE) from May to September 1998 and 1999a

\begin{tabular}{|c|c|c|c|c|c|c|c|c|c|c|}
\hline \multirow[b]{2}{*}{ Site } & \multirow[b]{2}{*}{ No. ${ }^{d}$} & \multirow[b]{2}{*}{ MWD $^{\mathbf{e}}$} & \multicolumn{4}{|c|}{ ME (h/day) $(\text { SEM })^{b}$} & \multicolumn{4}{|c|}{$\operatorname{MAE}(\mathbf{h} / \text { day })^{\mathbf{c}}$} \\
\hline & & & SkyBit & CART & FL & CFL & SkyBit & CART & FL & CFL \\
\hline Ames, IA & 272 & 8.6 & $-2.9(0.46)$ & $-2.7(0.31)$ & $-4.6(0.32)$ & $-1.4(0.33)$ & 9.0 & 5.8 & 6.6 & 5.7 \\
\hline Lewis, IA & 276 & 7.5 & $-0.2(0.44)$ & $0.2(0.30)$ & $-2.0(0.33)$ & $0.9(0.33)$ & 8.0 & 5.1 & 5.5 & 5.5 \\
\hline Nashua, IA & 270 & 8.0 & $-1.2(0.41)$ & $-0.4(0.31)$ & $-1.9(0.31)$ & $1.2(0.32)$ & 7.8 & 4.5 & 4.9 & 4.9 \\
\hline Sutherland, IA & 275 & 8.0 & $-1.6(0.42)$ & $-1.0(0.32)$ & $-2.4(0.33)$ & $0.8(0.33)$ & 7.8 & 5.1 & 5.5 & 5.3 \\
\hline Crawfordsville, IA & 275 & 8.0 & $-3.5(0.43)$ & $-2.5(0.28)$ & $-5.0(0.29)$ & $-1.8(0.28)$ & 8.3 & 5.0 & 6.1 & 4.9 \\
\hline Belleville, IL & 184 & 7.7 & $-4.7(0.49)$ & $-5.1(0.35)$ & $-7.0(0.33)$ & $-5.7(0.34)$ & 8.3 & 6.2 & 7.3 & 6.6 \\
\hline Bondville, IL & 208 & 9.9 & $-3.3(0.51)$ & $-1.2(0.32)$ & $-4.1(0.31)$ & $-1.0(0.31)$ & 9.1 & 4.6 & 5.9 & 4.5 \\
\hline Dixon Springs, IL & 209 & 9.2 & $-3.0(0.44)$ & $-1.1(0.33)$ & $-4.5(0.38)$ & $-1.3(0.38)$ & 8.1 & 4.6 & 6.2 & 5.0 \\
\hline Monmouth, IL & 208 & 7.4 & $-2.2(0.53)$ & $-1.7(0.36)$ & $-3.9(0.35)$ & $-0.8(0.36)$ & 8.0 & 5.3 & 5.8 & 5.2 \\
\hline St. Charles, IL & 204 & 8.6 & $-4.3(0.48)$ & $-4.9(0.33)$ & $-6.3(0.30)$ & $-3.1(0.34)$ & 8.5 & 6.4 & 7.0 & 5.7 \\
\hline Red Cloud, NE & 279 & 7.9 & $-2.0(0.45)$ & $-2.7(0.32)$ & $-4.1(0.32)$ & $-1.6(0.31)$ & 7.9 & 5.4 & 6.0 & 5.2 \\
\hline Gordon, NE & 283 & 8.5 & $-4.4(0.43)$ & $-5.5(0.34)$ & $-5.3(0.36)$ & $-3.0(0.36)$ & 8.1 & 7.0 & 7.1 & 6.2 \\
\hline O'Neill, NE & 228 & 6.5 & $-0.5(0.50)$ & $-1.4(0.36)$ & $-2.1(0.32)$ & $0.6(0.33)$ & 7.8 & 5.5 & 5.2 & 5.5 \\
\hline Sidney, NE & 283 & 6.4 & $-1.9(0.35)$ & $-2.4(0.30)$ & $-2.1(0.30)$ & $0.4(0.29)$ & 5.8 & 4.6 & 4.7 & 4.6 \\
\hline West Point, NE & 137 & 10.8 & $-3.3(0.57)$ & $-3.5(0.42)$ & $-5.4(0.42)$ & $-2.5(0.46)$ & 9.0 & 5.6 & 6.9 & 5.6 \\
\hline All 15 sites & 3,591 & 8.1 & $-2.5(0.12)$ & $-2.3(0.09)$ & $-3.9(0.09)$ & $-1.1(0.09)$ & 8.0 & 5.4 & 6.0 & 5.3 \\
\hline
\end{tabular}

a SkyBit = SkyBit wetness model, CART = CART/SLD/Wind model (14), FL = Fuzzy model, and CFL = an adjusted version of the Fuzzy model using a correction factor that increased the output of the Fuzzy model by $2.5 \%$.

${ }^{\mathrm{b}} \mathrm{ME}=\Sigma(\Sigma[$ estimated - measured $]) / \mathrm{N}$ and SEM $=$ standard error of the mean difference.

${ }^{\mathrm{c}} \mathrm{MAE}=\Sigma(\Sigma \mid$ estimated - measured $\mid) / \mathrm{N}$.

${ }^{\mathrm{d}}$ Number of 24-h periods included in the analysis.

${ }^{\mathrm{e}} \mathrm{MWD}=$ mean wetness duration (h/day) measured by sensors during study periods. 
FL model underpredicted LWD by $>2 \mathrm{~h}$ day $^{-1}$ at all sites except Nashua, IA. In contrast, the magnitude of MAE for the CFL model was similar to that for the CART and FL models. The SkyBit model forecasted LWD with considerably larger MAE $\left(8 \mathrm{~h} \mathrm{day}^{-1}\right)$ than the other models $(5$ to $6 \mathrm{~h} \mathrm{day}^{-1}$ ).

For days on which forecasted index values were $\leq 4$ for Melcast or $\leq 2$ for TOMCAST, index values obtained from sitespecific forecasts were similar to those obtained from measurements (Fig. 2A and C). However, as index values increased above these levels, forecasted values overestimated measured values by an increasing margin. On the other hand, the frequency of days with index values $>4$ for Melcast and $>2$ for TOM-CAST was low in the data set (Fig. 2B and D). LWD models sometimes predicted a disease-warning index of zero when an index derived from measurements was $>0$. For example, the SkyBit and FL models forecasted an index value of zero for both Melcast and TOM-CAST on about $60 \%$ of days. In contrast, the index value derived from weather measurements were zero on about 40 and $30 \%$ of days for Melcast and TOM-CAST, respectively.
Timing of forecasted spray dates was relatively accurate when cumulative disease-warning index values were derived by combining forecasted and measured weather data (Fig. 3). The CART and CFL models resulted in spray timing forecasts on the exact day for approximately $45 \%$ of the Melcast and TOM-CAST simulations. Forecast of spray advisories based on the SkyBit and FL models, however, tended to miss exact spray dates in a greater percentage of cases. When hindcast estimates rather than on-site measurements were combined with forecasts to calculate the cumulative index, accuracy of forecasting spray advisories on the exact date decreased considerably for all models (Fig. $4)$. Nevertheless, the CART model forecasted spray advisories within \pm 3 days for approximately $70 \%$ of Melcast and TOMCAST simulations. Accuracy of the CFL model in predicting disease-warning indexes was similar to that of the CART model.

\section{DISCUSSION}

This is the first report validating sitespecific forecasts of LWD for potential implementation in disease-warning sys- tems. Using forecasted weather data and LWD models in simulations of the Melcast and TOM-CAST warning systems, timing of action thresholds for fungicide sprays was predicted with comparable accuracy to predictions derived from weather measurements.

Integration of forecasted LWD into disease-warning systems could have an enormous impact on the practical implementation of disease-warning systems. Armed with 24-h forecasts, growers could apply control measures while it was still possible to drive spray equipment through a field or orchard. By preventing weather-related preemption of control measures, such a capability could reduce the risk of control failure when a disease-warning system is implemented (28). This important advantage should be weighed against the errors incurred in forecasting schemes.

Our results suggest, however, that the accuracy of site-specific forecasts needs to be improved in order to predict spray timing in practice. Forecasted air temperature of the SkyBit dataset was reasonably similar to measurements $\left(R^{2}=0.83\right)$. Truxall and Travis (28) also reported that the MASS model, a base model used to gener-
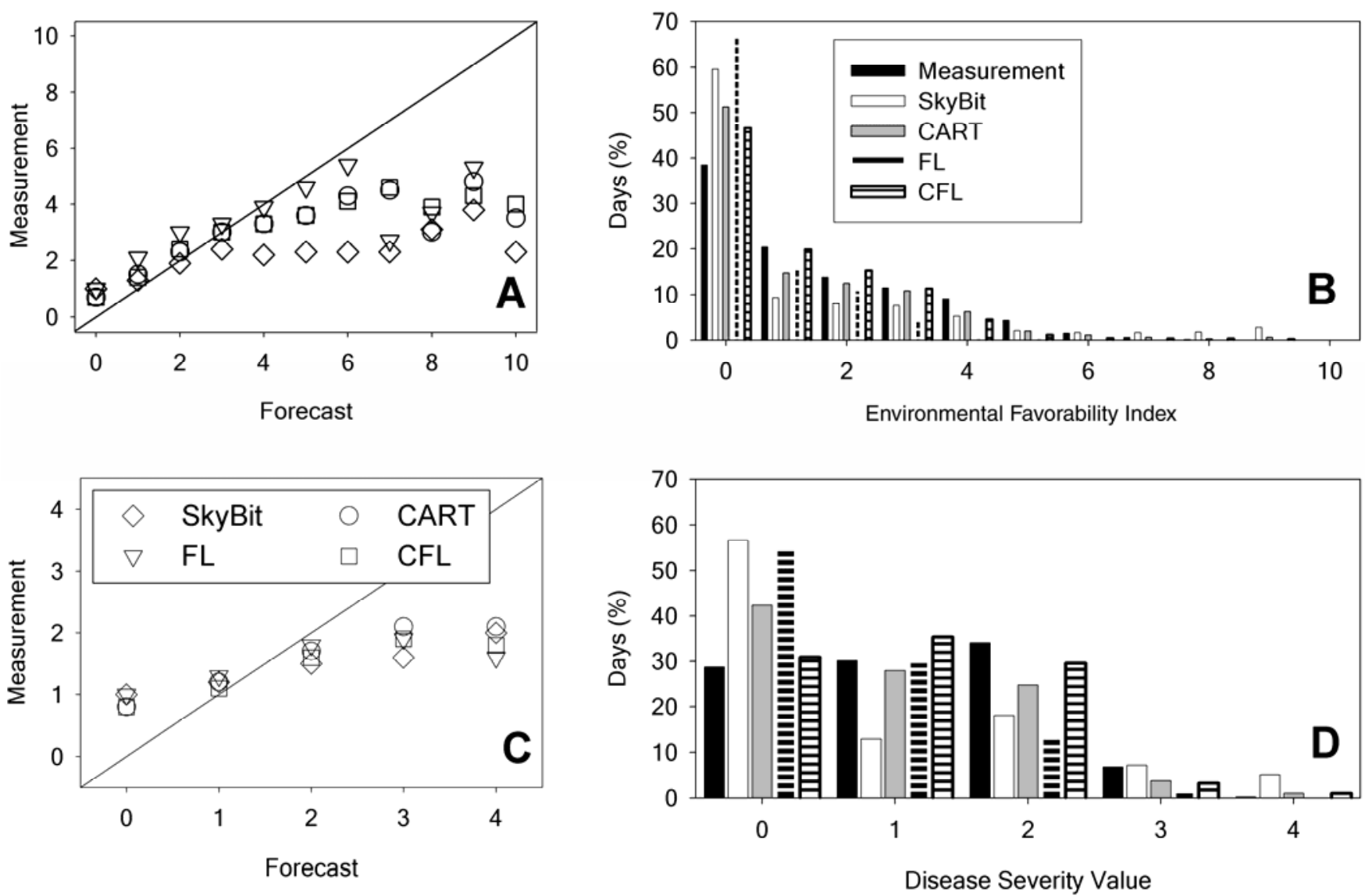

Fig. 2. Accuracy of forecasted disease-warning indices for Melcast (A and B) and TOM-CAST (C and D). A and C, Expected values of an individual disease-warning index forecast, calculated as follows: $\operatorname{Exp}(D W I)=\sum_{i=1}^{n} p(D W I=i) \cdot i$ where $p$ is the probability of an individual value of disease-warning index,

DWI a random variable on $\{0,1,2, \ldots, n\}$, and $n$ the largest value of DWI corresponding to either "environmental favorability indices" (EFI) and "disease severity values" (DSV). B and D, Number of days on which specific values of measured and forecasted disease-warning indexes for the Melcast (B) and TOM-CAST (D) disease warning systems occurred. 
ate weather data by SkyBit, forecasted air temperature reasonably accurately. Therefore, a disease-warning system based on air temperature (10) potentially could provide accurate prediction of spray advisories using the SkyBit dataset. In contrast, site-specific forecasts of $\mathrm{RH}$ contained substantial errors that were both systematic and random (Fig. 1). For example, RH was sometimes underpredicted when air tem-

perature was underpredicted, which is inconsistent with meteorological principles. Because RH is an essential input to LWD estimation models, this inconsistency could hamper efforts to improve the accuracy of a disease-warning system based on LWD estimates.

Accurate prediction of fungicide or bactericide spray timing requires acceptably accurate LWD estimates when no LWD
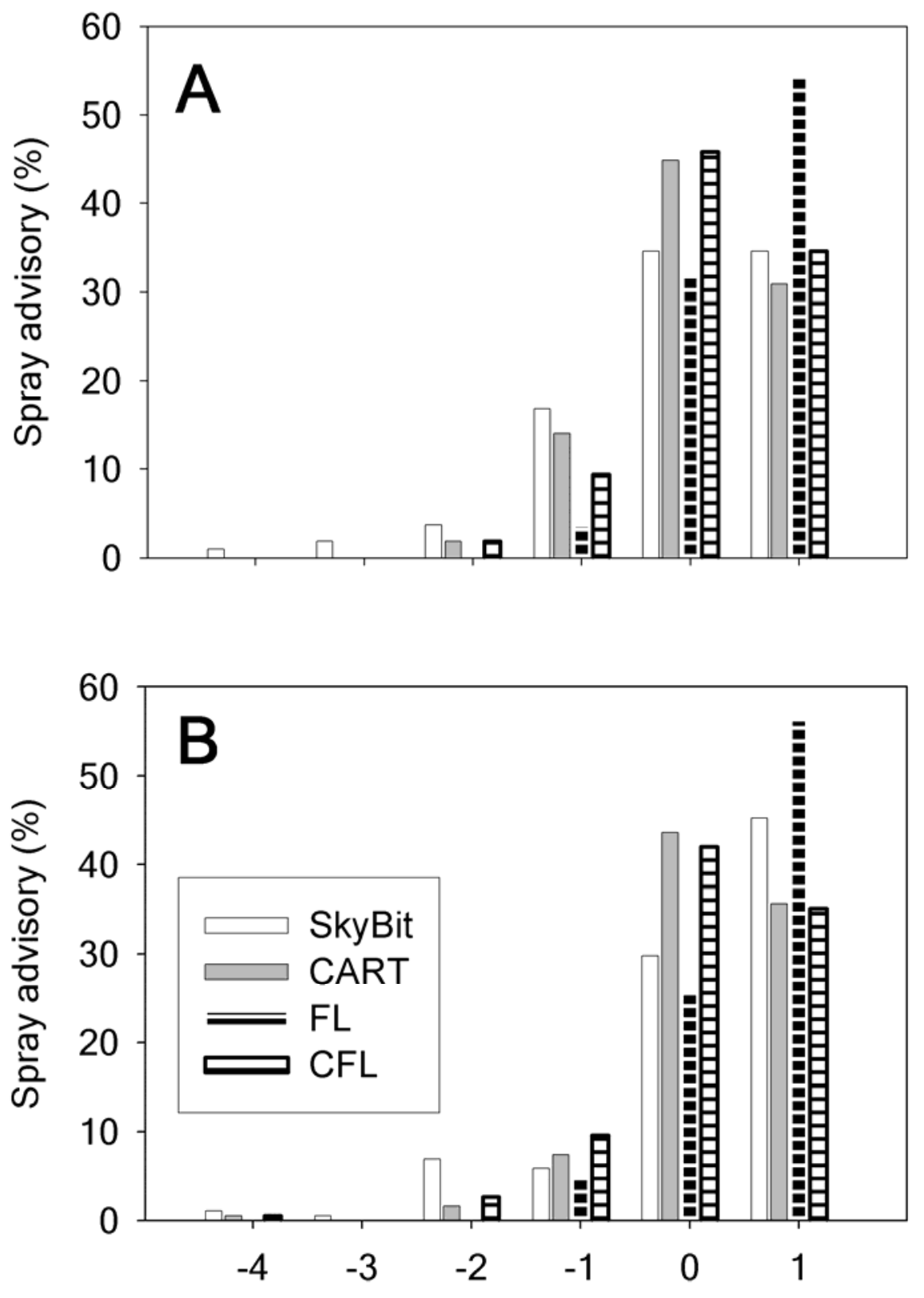

\section{Error (Forecast - Measurement) (day)}

Fig. 3. Accuracy of forecasted spray advisories for Melcast (A) and TOM-CAST (B) obtained by combining on-site weather measurements with 24-h forecasts of air temperature and leaf wetness duration (LWD). Error represents the difference, in days, between occurrence of action thresholds derived from weather measurements and those derived from weather forecasts that were applied only on action-threshold decision dates. In the forecast simulations, Melcast or TOM-CAST values were determined by weather measurements on all days until an action threshold was attained; on these decision dates alone, model-derived forecasts were applied. If a forecasted spray advisory occurred before the date that had been calculated solely by weather measurements, a negative value of error was depicted. If no spray advisory was forecasted on that date, the spray advisory forecast error was set equal to 1 . measurements are available. Our results showed that LWD models differed in their sensitivity to errors in site-specific forecasts. For example, the MAE of the SkyBit model was considerably larger $\left(8 \mathrm{~h} \mathrm{day}^{-1}\right)$ than that of the other models ( 5 to $\left.6 \mathrm{~h} \mathrm{day}^{-1}\right)$. The FL model underestimated LWD (Table 2 ), probably because underprediction of the RH input data led to errors in predicting water vapor pressure. In contrast, the CART model predicted LWD with reasonable accuracy $\left(-2.3 \mathrm{~h} \mathrm{day}^{-1}\right)$ using forecast weather data.

Using a correction factor, the CFL model substantially reduced the magnitude of LWD estimation errors by the FL model (Table 2). The two major input variables of the FL model, vapor pressure deficit (VPD) and estimated net radiation, are closely related to accuracy of estimated water vapor pressure. Therefore, accuracy of the FL model is dependent on accuracy of water vapor pressure estimates derived from RH and air temperature. In the fuzzy logic system on which the FL model is based, so-called "fuzzy" rules are evaluated using linguistic terms (e.g., "high" at the degree of 0.5 , associated with their input variables). When an input value to a fuzzy logic model contains small errors, it is possible to prevent its error from affecting the accuracy of model outcomes. When the magnitude of an input error becomes large, however, the linguistic term converted from the input value represents an erroneous state of the variable, resulting in inaccurate evaluation of logic statements. It is likely that underestimated site-specific forecasts of RH caused such errors in the FL model, which caused consistent underprediction of LWD. The correction factor in the CFL model adjusted these errors to predict LWD accurately.

The correction factor was not able to reduce the magnitude of MAE for the CFL model, however. This outcome suggests that the effectiveness of an empirically derived correction factor may be limited unless physical or meteorological conditions support its use. When a correction factor was applied to the CFL model during the dry season in northwestern Costa Rica, its application was justified on meteorological principles, because the extremely dry climate caused overestimation of radiation in the FL model, resulting in underestimation of LWD (15). In the present study, however, the site-specific weather forecasts contained not only systematic errors (i.e., those whose direction conformed with meteorological principles) but also random (i.e., unpredictable) errors that hampered improvement of LWD forecast using the correction factor. Alternative approaches to current spatial interpolation or temporal extrapolation schemes are needed to reduce those random errors and thereby improve LWD prediction.

In this study, LWD measurements made over turfgrass were extrapolated to crop 
canopies. Using measurements over turfgrass as a surrogate for measurements in nearby crop canopies offers practical advantages in that sensors and data-loggers may be more accessible and less threatened by farm machinery operations than in crop fields. In at least some temperatezone climates, the duration of dew periods is quite similar at the top of the canopy in fields of several types of cultivated plants, including turfgrass. This is because radiative cooling, which is likely to result in dew formation on clear and calm nights, is unimpeded at the top of crop canopies. In many temperate-zone climates, furthermore, dew periods constitute the majority of wet hours during the growing season $(7,14)$. In field studies, Sentelhas et al. (27) found that LWD measured at $0.3 \mathrm{~m}$ over turfgrass was highly correlated with LWD at the top of apple, maize, coffee, and grape canopies in Brazil, Iowa, and Ontario. In these trials, LWD at the top of the crop canopy equaled or exceeded LWD at lower canopy positions. These results suggest that, in climatic zones similar to those studied, LWD measured over turfgrass is suitable as a surrogate for crop canopies for implementation of disease-warning systems because it closely estimates LWD at the top of crop canopies and provides a conservative estimate of LWD elsewhere in the crop canopy.

The CART and CFL models appeared to be the most promising for applying forecasted LWD data to disease-warning systems. Expected values of forecasted indexes were close to the actual values on most days (Fig. 2), which suggests that these models possess low sensitivity to input errors when LWD estimates derived from site-specific forecasts are used as inputs to a disease-warning system. In Melcast and TOM-CAST simulations, spray dates forecasted by both LWD models were reasonably comparable to those derived from measurements. However, to confirm that the models can operate these warning systems effectively, field validation experiments that include crop disease assessment are needed at multiple sites over multiple years. In particular, validation of the CFL model is needed because the correction factor in the CFL model was obtained from a training process using the same hindcast dataset that drove simulation of these warning systems.

At relatively high values of warningsystem indexes, the models tended to overestimate risk. This overprediction was greatest for the highest risk ratings (Fig. 2). For both Melcast and TOM-CAST, overestimation of warning-system indexes occurred primarily during periods of extended wetness $(>12 \mathrm{~h})$. It is likely that overestimated site-specific forecasts of rainfall during daytime caused such errors, because such an error would result in predicting high humidity all day long and, in turn, overestimating LWD. The fact that such days occurred quite infrequently in the data set we analyzed suggests that these errors might not bias the operation of Melcast or TOM-CAST severely in Iowa, Illinois, and Nebraska. However, in regions where growing seasons included more "high-risk" days, the models' overestimation errors would be expected to further bias operation of the warning systems. In contrast, use of the models in TOM-CAST and Melcast tended to underpredict risk when the measured value of EFI or DSV was 1 . These errors are likely to have been caused by underestimation of RH, which in turn resulted in underprediction of LWD.

To achieve accurate site-specific forecasts of spray timing in practice, it is important to improve the accuracy of hindcast estimates of weather variables. Although site-specific estimates can reduce or eliminate the need for growers to monitor weather conditions in their fields, it is difficult to obtain accu-
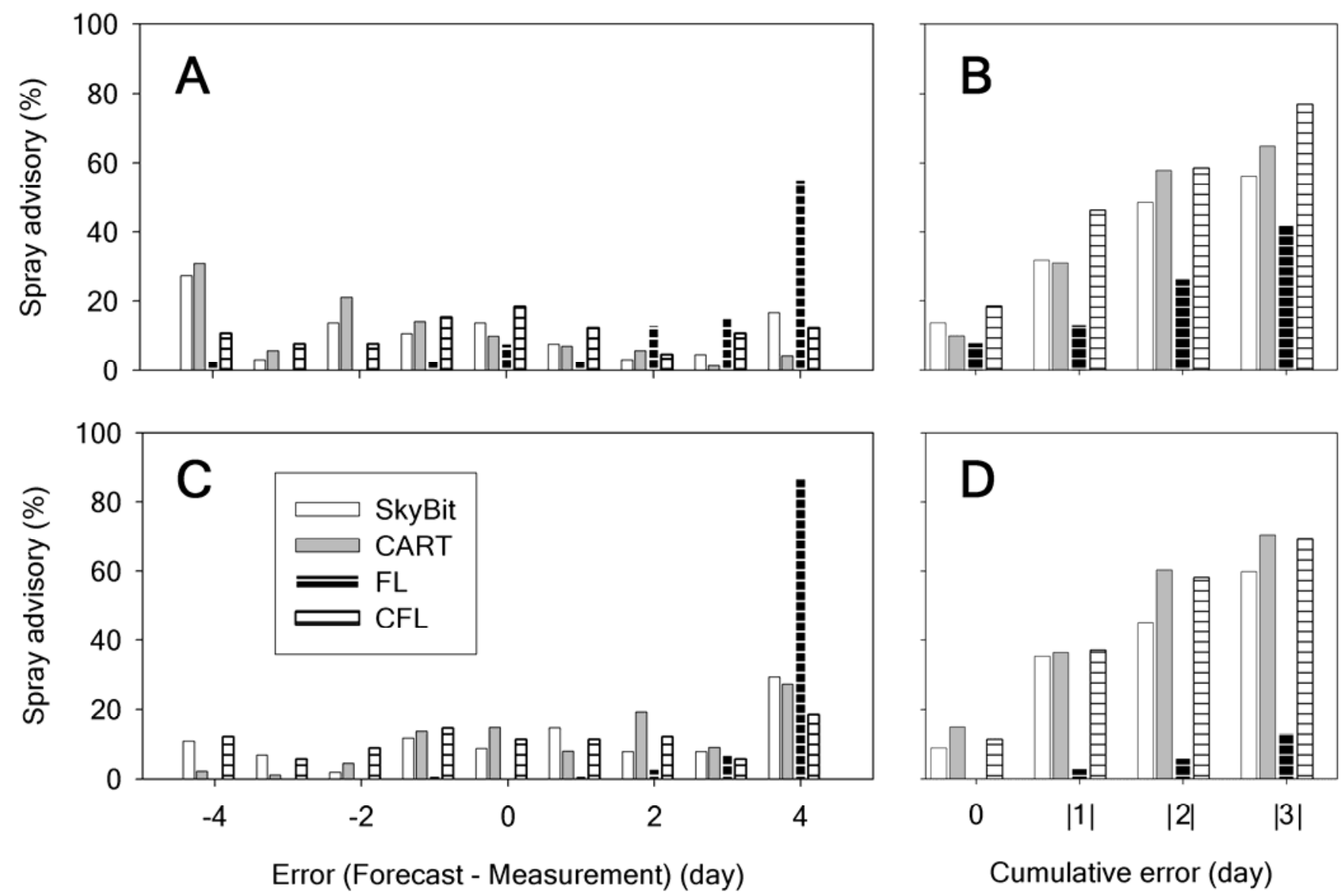

Fig. 4. Accuracy of forecasted spray advisories for Melcast (A and B) and TOM-CAST (C and D) obtained by combining model-estimated hindcast weather data with 24-h forecasts of air temperature and leaf wetness duration (LWD). A and C, Frequency distribution of errors in forecasting the date of occurrence of a fungicide-spray advisory. B and D, Range of cumulative error in timing of spray advisories. Errors represent difference (days) between spray dates obtained from on-site weather measurements and model-derived forecasts. 
rate estimates of LWD and disease-warning indices when hindcast estimates are inaccurate. Furthermore, prediction of a spray advisory requires accumulation of diseasewarning index values derived from those estimates over a number of consecutive days, which could cause accumulation of estimation errors.

Despite these limitations, our results suggest that the CART and CFL models can provide LWD predictions accurately enough to implement disease-warning systems (Figs. 3. and 4). The CART model required no correction to make relatively accurate predictions of LWD and implement diseasewarning systems. Although accuracy of the CFL model could be affected by the fact that its correction factor resulted from a training process based on the same hindcast data set used in simulation trials (Fig. 4), the correction factor minimized errors for all the subsets of the training set, suggesting that it also may reduce errors caused by sitespecific estimation of weather data under different sets of weather conditions. For new regions or sites, however, it would be necessary to validate the accuracy of the CFL model when hindcast estimates were used as inputs.

Site-specific weather data are available for any location in North America via readily accessible commercial services, so the CART and CFL models may be broadly applicable in disease-warning systems that use LWD as an input. With further testing, these models may help to warn growers in North America, and eventually in other regions of the world (15), about impending warning-system action thresholds at least $24 \mathrm{~h}$ in advance.

\section{ACKNOWLEDGMENTS}

We thank Ken Hubbard of the High Plains Climate Center, Lincoln, NE, Steve Hollinger of the Illinois State Water Survey, Champaign, IL, and Kent Barns of Iowa State University for their help in acquiring on-site weather data, and Ken Koehler of Iowa State University for advice and assistance in data archiving.

\section{LITERATURE CITED}

1. Chtioui, Y., Francl, L. J., and Panigrahi, S. 1999. Moisture prediction from simple micro- meteorological data. Phytopathology 89:668672.

2. Davis, D. R., and Hughes, J. E. 1970. A new approach to recording the wetting parameter by the use of electrical resistance sensors. Plant. Dis. Rep. 54:474-479.

3. Francl, L. J., and Panigrahi, S. 1997. Artificial neural network models of wheat leaf wetness. Agric. For. Meteorol. 88:57-65.

4. Gillespie, T. J., and Barr, A. 1984. Adaptation of a dew estimation scheme to a new crop and site. Agric. For. Meteorol. 31:289-295.

5. Gillespie, T. J., and Kidd, G. E. 1978. Sensing duration of leaf moisture retention using electrical impedance grids. Can. J. Plant. Sci. 58:179-187.

6. Gleason, M. L. 2001. Disease-warning systems. Pages 367-370 In: Encyclopedia of Plant Pathology, Vol. I. John Wiley \& Sons, New York.

7. Gleason, M. L., Parker, S. K., Pitblado, R. E., Latin, R. X., Speranzini, D., Hazzard, R. V., Maletta, M. J., Cowgill, W. P., Jr., and Biederstedt, D. L. 1997. Validation of a commercial system for remote estimation of wetness duration. Plant Dis. 81:825-829.

8. Gleason, M. L., Taylor, S. E., Loughin, T. M., and Koehler, K. J. 1994. Development and validation of an empirical model to estimate the duration of dew periods. Plant Dis. 78:1011-1016.

9. Huber, L., and Gillespie, T. J. 1992. Modeling leaf wetness in relation to plant disease epidemiology. Annu. Rev. Phytopathology 30:553577.

10. Johnson, K. B., Stockwell, V. O., and Sawyer, T. L. 2004. Adaptation of fire blight forecasting to optimize the use of biological controls. Plant Dis. 88:41-48.

11. Kaplan, M. L., Zack, J. W., Wong, V. C., and Tucillo, J. J. 1982. Initial results from an atmospheric simulation system and comparison with an AVE-SESAME I data set. Mon. Weather. Rev. 110:1564-1590.

12. Kelley, J. G. W., Russo, J. M., Eyton, J. R., and Carlson, T. N. 1988. Mesoscale forecasts generated from operational numerical weatherprediction model output. Bull. Am. Meteorol. Soc. 69:7-15.

13. Kim, K. S., Taylor, S. E., and Gleason, M. L. 2004. Development and validation of a leaf wetness model using a fuzzy logic system. Agric. For. Meteorol. 127:53-64.

14. Kim, K. S., Taylor, S. E., Gleason, M. L., and Koehler, K. J. 2002. Model to enhance sitespecific estimation of leaf wetness duration. Plant Dis. 86:179-185.

15. Kim, K. S., Taylor, S. E., Gleason, M. L., Villalobos, R., and Arauz, L. F. 2005. Estimation of leaf wetness duration using empirical models in northwestern Costa Rica. Agric.
For. Meteorol. 129:53-67.

16. Latin, R., and Egel, D. 2001. Melcast melon disease forecaster. Bulletin BP-64-W, Purdue University, West Lafayette, IN.

17. Lau, Y. F., Gleason, M. L., Zriba, N., Taylor, S. E., and Hinz, P. N. 2000. Effects of coating, deployment angle, and compass orientation on performance of electronic wetness sensors during dew periods. Plant Dis. 84:192-197.

18. Llorente, I., Vilardell, P., Bugiani, R. Gherardi, I., and Montesinos, E. 2000. Evaluation of BSPcast disease-warning system in reduced fungicide use programs for management of brown spot of pear. Plant Dis 84:631-637.

19. Madden, L. V., Ellis, M. A., Lalancette, N., Hughes, G., and Wilson, L. L. 2000. Evaluation of a disease-warning system for downy mildew of grapes. Plant Dis. 84:549-554.

20. Madeira, A. C., Kim, K. S., Taylor, S. E., and Gleason, M. L. 2002. A simple cloud-based energy balance model to estimate dew. Agric. For. Meteorol. 111:55-63.

21. Magarey, R. D.1999. A theoretical standard for estimation of surface wetness duration in grape. Ph.D. dissertation, Cornell University, Ithaca, NY.

22. Pedro, M. J., and Gillespie, T. J. 1982. Estimating dew duration. II. Utilizing standard weather station data. Agric. Meteorol. 25:297310.

23. Pitblado, R. E. 1988. The development of a weather-timed fungicide spray program for field tomatoes. Can. J. Plant Pathol. 10:371.

24. Potratz. K. J., Gleason, M. L., Hockmuth, M. L., Parker, S. K., and Pearston, G. A. 1994. Testing the accuracy and precision of wetness sensors in a tomato field and on turfgrass. J. Iowa Acad. Sci. 101:56-60.

25. Poysa, V., Brammall, R. A., and Pitblado, R. E. 1993. Effects of foliar fungicide sprays on disease and yield of processing tomatoes in Ontario. Can. J. Plant Sci. 73:1209-1215.

26. Rao, P. S., Gillespie, T. J., and Schaafsma, A. W. 1998. Estimating wetness duration on maize ears from meteorological observations. Can. J. Soil. Sci. 78:149-154.

27. Sentelhas, P. C., Gillespie, T. J., Batzer, J. C., Gleason, M. L., Monteiro, J. E. B. A., Pezzopane, J. R. M., and Pedro, M. J., Jr. 2005 Spatial variability of leaf wetness duration in different crop canopies. Int. J. Biometeorol. 49:363-370.

28. Shtienberg, D., and Elad, Y. 1997. Incorporation of weather forecasting in integrated biological-chemical management of Botrytis cinerea. Phytopathology 87:332-340.

29. Truxall, D. L., and Travis, J. W. 1994. Analysis of the accuracy of predicted weather data on the impact of apple disease management. (Abstr.) Phytopathology 84:1125. 